\title{
Transmission electron diffraction from nanoparticles, nanowires and thin films in an SEM with conventional EBSD equipment*
}

\author{
R.H. Geiss, R.R. Keller, and D.T. Read \\ Materials Reliability Division, National Institute of Standards and Technology, Boulder, CO
}

We describe a new scanning electron microscope (SEM) method for obtaining and analyzing the crystallographic structure and orientation of nanoparticles and ultrathin films with conventional electron backscatter diffraction (EBSD) equipment. Thinned electron-transparent samples and nanoparticles or nanowires suspended on transparent carbon film substrates were positioned immediately below the pole piece of the SEM by use of a custom made holder. This allowed electron diffraction patterns in transmission from nanodimensioned samples to be captured in an SEM by a conventional EBSD camera obviating the need for a transmission electron microscope (TEM). The resulting diffraction patterns were displayed with EBSD software on the computer screen. In this fashion, we obtained diffraction patterns from nanoparticles as small as $20 \mathrm{~nm}$ in diameter and from nanowires with widths as small as $30 \mathrm{~nm}$. Patterns were also obtained from free-standing thin film metal samples sandwiched in copper grids and from thin metal samples deposited on silicon nitride window substrates. In these samples, orientation mapping was facilitated by scanning the electron beam in the same manner that is used in reflection EBSD. By detecting the spatial variation in high-angle transmitted-electron scattering from a nanoparticle or thin free-standing film, we obtained improvements in pattern contrast, signal to noise ratio, and lateral spatial resolution compared to the same factors observed with conventional electron backscatter diffraction (EBSD). The correlation between transmitted energy spread and image contrast can be qualitatively explained with the Monte Carlo simulations shown in figure 1 . The improvements are thought to be due in part to a strongly reduced contribution from diffuse scattering. The self-energyfiltering capacity of electron transmission through nanoscale specimens can result in usable SEM-based diffraction patterns from individual grains having lateral dimension as small as approximately $10 \mathrm{~nm}$. In figure 2, we show an example where good patterns were obtained in transmission from a $40 \mathrm{~nm}$ thick nickel film deposited on a silicon nitride substrate. We were not able to obtain well-defined EBSD patterns from this sample with the conventional EBSD reflection mode. In figure 3, we give examples of transmission electron diffraction patterns from iron cobalt nanoparticles (diameter of $10 \mathrm{~nm}$ ), in figure 4, from $\mathrm{Al}_{2} \mathrm{O}_{3}$ nanoparticles (diameter $20 \mathrm{~nm}$ ), and in figure 5, from $\mathrm{GaN}$ nanowires with $30 \mathrm{~nm}$ to $50 \mathrm{~nm}$ wide hexagonal cross-sections. In figure 6 , we show an orientation map from transmission electron diffraction patterns from an aluminum film, $150 \mathrm{~nm}$ thick. Finally, we have observed that for diffraction patterns formed by transmitted electrons, the quality of the specimen surface has a much less significant effect on pattern contrast than it does for EBSD patterns formed by reflected electrons.

* Contribution of the U.S. Department of Commerce, National Institute of Standards and Technology. Not subject to copyright in the U.S.A. 


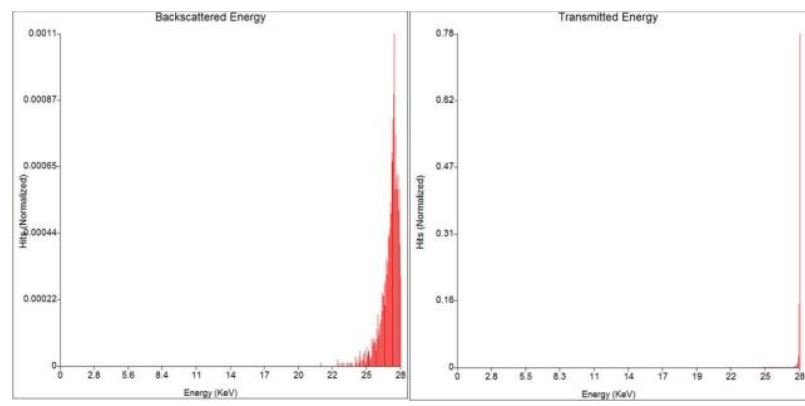

Figure 1. Monte Carlo simulations for a $40 \mathrm{~nm}$ Ni film on $2.5 \mathrm{~nm}$ Ta on a $50 \mathrm{~nm}$ silicon nitride substrate, with a mean Ni grain diameter of $14.5 \mathrm{~nm}$. Beam energy $=28 \mathrm{keV}$.

(a) Backscattered electron energy spectrum.

(b) Transmitted electron energy spectrum.

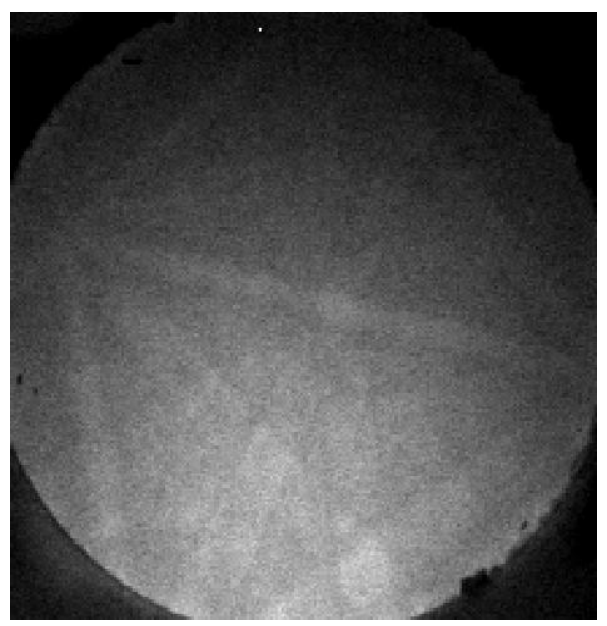

Figure 3. Transmission pattern from $10 \mathrm{~nm}$ diameter nanoparticle of iron cobalt.

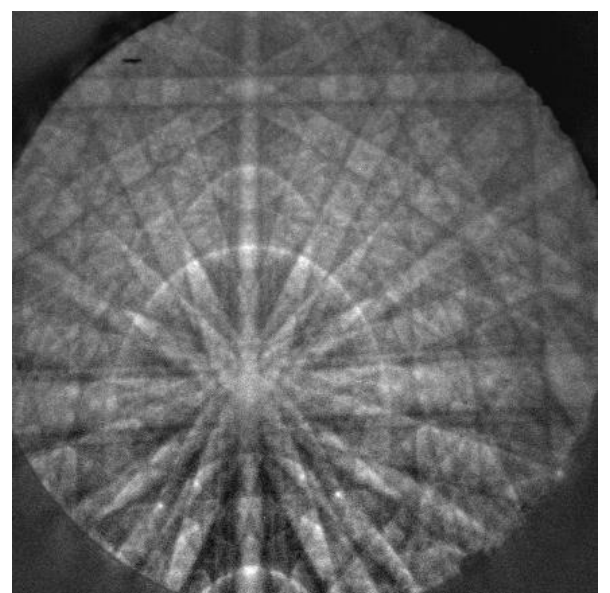

Figure 5. Transmission pattern from $30 \mathrm{~nm}$ diameter $\mathrm{GaN}$ nanowires.

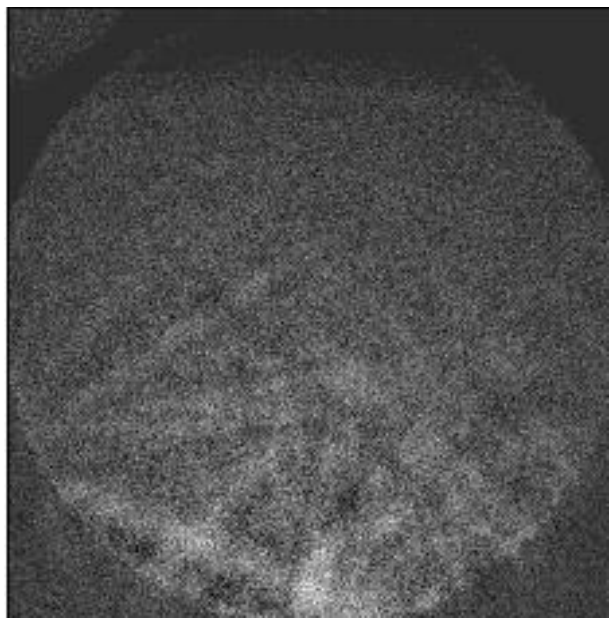

Figure 2. Transmission pattern from single grain in a $40 \mathrm{~nm}$ Ni film on $2.5 \mathrm{~nm}$ Ta deposited on a $50 \mathrm{~nm}$ silicon nitride substrate.

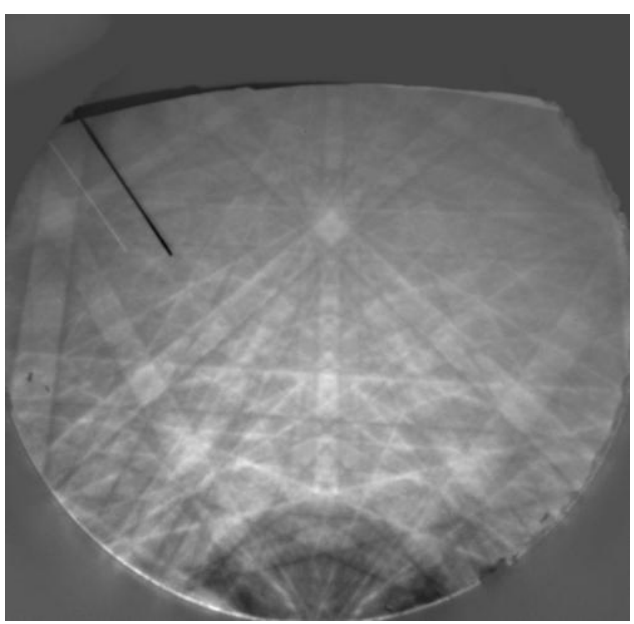

Figure 4. Transmission pattern from $20 \mathrm{~nm}$ diameter $\mathrm{Al}_{2} \mathrm{O}_{3}$ nanoparticles.

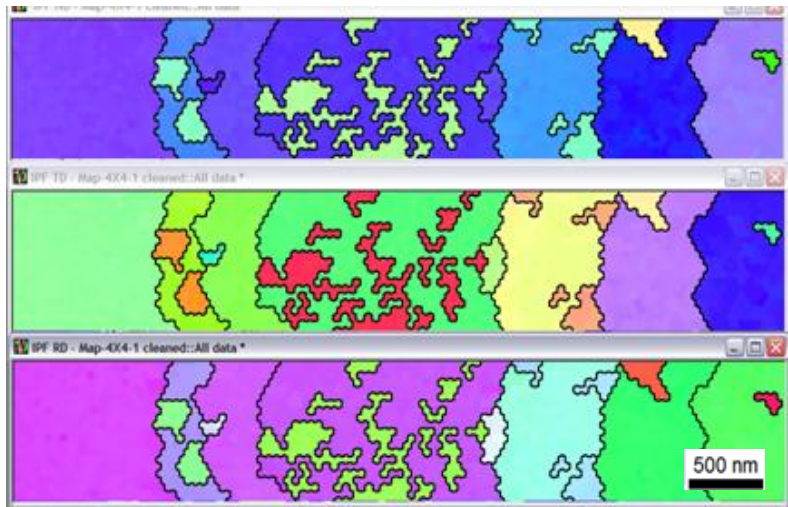

Figure 6. Inverse pole figure orientation maps in three orthogonal orientations from an aluminum film of thickness $150 \mathrm{~nm}$. 